\title{
Exploring the impact of core expansion on the vortex distribution in superconducting-normal-metal hybrid nanostructures
}

\author{
Ritika Panghotra, ${ }^{1, *}$ Matias Timmermans, ${ }^{1,2}$ Cun Xue, ${ }^{3}$ Bart Raes, ${ }^{1}$ Victor Moshchalkov, ${ }^{1}$ and Joris Van de Vondel ${ }^{1}$ \\ ${ }^{1}$ Laboratory of Solid State Physics and Magnetism, Department of Physics and Astronomy, \\ KU Leuven, Celestijnenlaan 200D, B-3001 Leuven, Belgium \\ ${ }^{2}$ Catalan Institute of Nanoscience and Nanotechnology (ICN2), CSIC and BIST, Campus UAB, Bellaterra, 08193 Barcelona, Spain \\ ${ }^{3}$ School of Mechanics, Civil Engineering and Architecture, and MIIT Key Laboratory of Dynamics and Control of Complex Systems, \\ Northwestern Polytechnical University, Xi'an 710072, People's Republic of China
}

(Received 26 March 2019; revised manuscript received 19 June 2019; published 29 August 2019)

\begin{abstract}
The superconducting condensate in a bilayer normal-metal-superconducting film has a nonuniform dependence throughout the layer. For sufficiently thin normal metallic films, the superconducting correlations induced by the contacting superconducting layer result in the formation of a minigap and the characteristic length scales governing the response of the condensate will be different. In this work we use scanning tunneling spectroscopy to visualize the vortex states as a function of the applied magnetic field in a Au-MoGe nanostructure. By comparing the obtained zero-bias conductance maps with time-dependent Ginzburg-Landau simulations, we directly confirm that the observed vortex distributions can only be explained by taking into account the impact of the normal metallic layer. We illustrate this impact on the vortex state for two lithographically fabricated mesoscopic bilayer structures containing an identical antidot array but having different lateral sizes.
\end{abstract}

DOI: 10.1103/PhysRevB.100.054519

\section{INTRODUCTION}

The ability to combine superconducting materials with other (metallic, magnetic, semiconducting, and two-dimensional) materials, opens a variety of possibilities to create novel hybrid structures with unprecedented functionalities [1-4]. The particular case of a bilayer structure, combining a normal and superconducting thin film, results in the existence of superconducting correlations in the metal, characterized by the formation of a minigap, while the superconducting order parameter inside the superconducting material will be partially suppressed [5-7]. In addition, the coherence length will drastically expand inside the diffusive metal [8]. This resulting expansion of the vortex core will have an important impact on the observed vortex distributions because (i) the interaction between vortices will be nonuniform throughout the bilayer and (ii) the interaction of the vortex distribution with a nanostructured environment is dependent on the ratio between the characteristic length scale of the condensate, i.e., the coherence length, and the actual size of the nanostructure [9-11].

Following the motto "seeing is believing," we find that scanning probe techniques are the tools at hand to reveal the supercurrent distribution and the spatial variations of the superconducting condensate in nanostructured superconductors. Whereas the magnetic field can be probed by field-sensitive techniques such as scanning Hall probe microscopy [12], scanning SQUID microscopy, magneto-optical imaging [13], and Bitter decoration [14], both the spatial variation of the superconducting density of states and the supercurrent

*ritika.panghotra@kuleuven.be can be probed directly by scanning tunneling spectroscopy (STS) [15]. While the latter technique can measure direct variations in the condensate arising on a length scale of the socalled coherence length, $\xi$, with atomic resolution, the former techniques can infer only indirect information concerning the current distribution [16-18] by picking up the resulting variations in the magnetic field arising on a characteristic length scale of the penetration depth, $\lambda$. The spatial resolution of these techniques is typically limited by the size of the magnetic field probe (>50 nm) [19]. Consequently, to study the condensate in nanostructured superconductors, the superior spatial resolution of STS makes it the preferential tool. However, despite the numerous successes of STS to study the superconducting condensate [20,21], only a handful of experiments have been performed on lithographically defined mesoscopic samples [18,22,23]. This is due to the extreme sensitivity of the STS technique to the sample surface quality, traditionally limiting this technique to cases where the sample surface can be in situ prepared (i.e., under UHV conditions) or in situ cleaved [20,21,24-26].

In this work, we present STS measurements on two mesoscopic squares $(\xi<$ dimension squares $<\lambda)$ of different size, containing an identical square array of circular antidots. In order to enable STS measurements on these superconducting nanostructures of molybdenum germanium (MoGe), they are protected by a thin gold ( $\mathrm{Au}$ ) capping layer [18]. As a result, a nanostructured superconducting-normal-metal bilayer is created. The STS measurements directly show the presence of a minigap in the $\mathrm{Au}$ layer [27,28], which allows us to directly observe the vortex distributions in the mesoscopic squares as a function of the applied magnetic field [8]. To understand the observed vortex distributions in both nanostructures, 

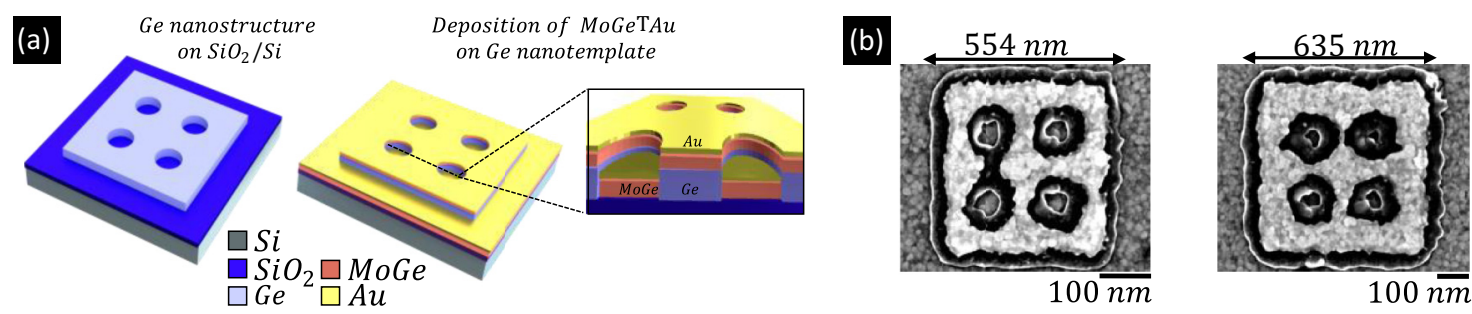

FIG. 1. (a) The two panels illustrate schematically the fabrication process of the mesoscopic squares containing a square antidot lattice by subsequent deposition of MoGe and $\mathrm{Au}$ on top of a Ge nanostructure. We also provide a schematic cross-sectional image along a cut through the antidot array. (b) Scanning electron microscopy micrographs of the investigated samples. The dark black color indicates the MoGe layer below the Au layer. The latter can be clearly identified by its typical bright granular structure. The Au film is slightly smaller than the MoGe structure due to incomplete wetting.

we make a direct comparison between the experimental observations and the numerical simulations performed within the Ginzburg-Landau model. This comparative study proves that the observed vortex distributions are the result of minimizing both the kinetic energy of the supercurrents and the loss in condensation energy needed to create a vortex [20]. Moreover, a good quantitative agreement is only achieved for both samples if we incorporate the impact of the metallic top layer on the overall superconducting properties by using an effective coherence length exceeding the value obtained for the MoGe thin film. This can be explained by the observed vortex core expansion throughout the normal layer of the mesoscopic structure [8]. These findings, demonstrating STS on lithographical fabricated nanostructured samples, open the pathway to exploit the unmatched resolution of STS to explore and characterize novel superconducting devices.

\section{SAMPLE FABRICATION AND CHARACTERIZATION}

The mesoscopic superconducting square geometries studied in this work by STS are shown schematically in Fig. 1(a). Both squares contain four antidots having a radius of $r \sim$ $25 \mathrm{~nm}$ arranged in a square lattice having a center-tocenter distance of $d \sim 200 \mathrm{~nm}$. One sample has a size of $a_{A} \sim 554 \mathrm{~nm}$ (sample A) and a second sample has a size of $a_{B} \sim 635 \mathrm{~nm}$ (sample B). As a consequence, the two sample geometries have an identical antidot lattice, but the outer rim surrounding the antidot lattice is approximately twice as wide for sample B.

The process to fabricate these mesoscopic structures with a high-quality surface, enabling exploration with STS, follows a similar procedure as outlined in Ref. [18] and is schematically shown in Fig. 1(a). This particular fabrication process minimizes the presence of unwanted contamination from the lithography process and guarantees a high surface quality, beneficial for STS measurements. In a first step, a nanotemplate of germanium is fabricated on top of a bare $\mathrm{Si} / \mathrm{SiO}_{2}$ substrate by a standard $e$-beam lithography process followed by subsequent deposition of a 30-nm-thick Ge film. In a second step, a 17-nm-thick molybdenum germanium layer $\left(\mathrm{Mo}_{79} \mathrm{Ge}_{21}\right)$ and a 5-nm-thick Au capping layer are deposited in situ on the predefined Ge nanotemplate using pulsed laser deposition. The Au capping layer is necessary to prevent oxidation of the MoGe layer and guarantees good tunnel conditions. The chosen thicknesses of the consecutive layers assure that (i) the MoGe-Au layer on top of the $\mathrm{Ge}$ square is disconnected from the layer on top of the bare substrate and (ii) the chosen thickness of the Au layer, a diffusive metal, assures that the proximity-induced minigap is well resolvable by STS [8].

Note that we have selected MoGe as the superconducting medium. MoGe is a type-II superconductor well known for its weak intrinsic pinning and low surface roughness. The rms value of the surface roughness for the combined MoGe-Au film is about $1.2-3 \mathrm{~nm}$ as determined from atomic force microscopy measurements. As such, this material is well suited to study the vortex configurations governed by confinement effects, artificial pinning centers, and vortex-vortex interactions, rather than dominated by strong random intrinsic pinning sites. A plain reference film of a MoGe-Au bilayer, coevaporated with the samples used for the STS experiments, was used to characterize the superconducting properties via standard transport measurements. These measurements indicate that the bilayer has a critical temperature of $T_{c}=5 \mathrm{~K}$ as determined by a $50 \%$ of the normal state resistance criterion. From a measurement of the superconductor-normal-phase boundary, a coherence length of $\xi(0) \sim 6.72 \mathrm{~nm}$ at zero temperature and a critical field of $H_{c 2}(T=0) \sim 7.26 \mathrm{~T}$ are estimated. The effective penetration depth, $\Lambda_{\mathrm{eff}}=\lambda^{2} / t_{\mathrm{MoGe}} \sim$ $14 \mu \mathrm{m}$, where $\lambda$ is the London penetration depth taken from Ref. [29] exceeds the sample dimensions, indicating the samples are in the mesoscopic regime.

\section{EXPERIMENTAL DETAILS AND THEORETICAL FORMALISM}

The STS measurements that reveal the spatial variation of the condensate were conducted using a commercial $\mathrm{He}^{3}$ system of Attocube having a base temperature of $\mathrm{T}=500 \mathrm{mK}$. A wire-cut Pt-Ir tip was used to perform the tunneling experiments. All the measurements were performed at the base temperature by increasing an external magnetic field, applied perpendicular to the sample surface, after preparing the sample by a zero-field-cooling (ZFC) procedure. This particular preparation of the vortex state serves a twofold purpose: (i) experimentally it is faster to obtain consecutive images at different magnetic field values (i.e., no need to increase temperature above $T_{c}$ ), and (ii) the induced vortex state will be more sensitive to the different sample edges of samples A and $\mathrm{B}$. 


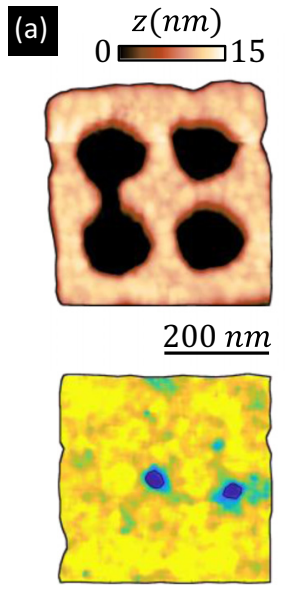

$320 m T$
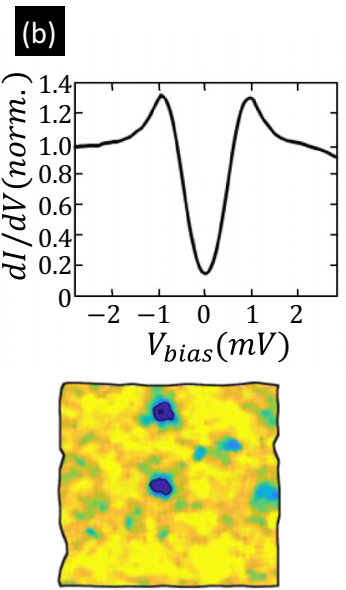

$330 m T$ (c)
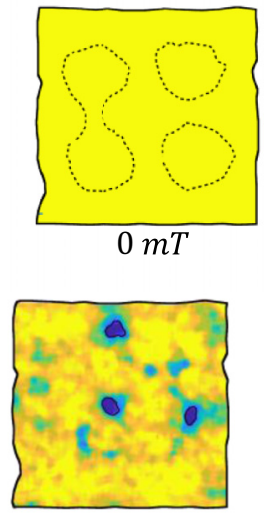

$340 m T$

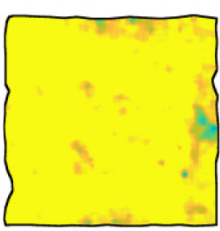

$220 m T$

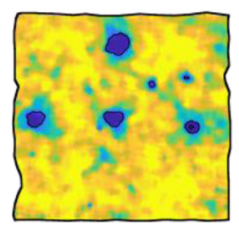

$350 m T$

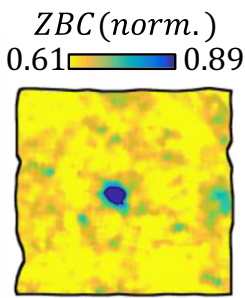

$310 m T$

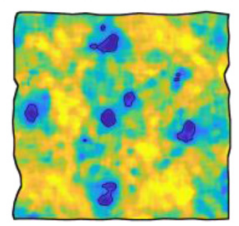

$400 m T$

FIG. 2. (a) A constant current topographic STM image of sample A obtained at a fixed tunnel current of $I_{t}=100 \mathrm{pA}$, using a bias voltage of $V_{\text {bias }}=5 \mathrm{mV}$. (b) A normalized local tunneling conductance spectrum, $d I / d V\left(V_{\text {bias }}\right.$ ), obtained at $T=0.5 \mathrm{~K}$ and $H=0 \mathrm{mT}$ (using as the setpoint a tunnel current of $I_{t}=100 \mathrm{pA}$ at a bias voltage of $V_{\text {bias }}=5 \mathrm{mV}$ ). The tunneling conductance is normalized to the value at $V_{\text {bias }}=3$ $\mathrm{mV}$. (c) Normalized ZBC maps obtained by STS measurements (normalized to the value at $V_{\text {bias }}=3 \mathrm{mV}$ ). The different images show a subset of the measured ZBC maps for increasing magnetic field values after preparing the sample by a zero-field-cooling procedure and increasing the field in steps of $H=10 \mathrm{mT}$. The black solid contour lines correspond with a $\mathrm{ZBC}=0.83$. The dotted black line in the panel for $H=0 \mathrm{mT}$ indicates the position of the antidots.

In order to understand the observed magnetic field dependence of the condensate and the observed vortex configurations, we performed numerical simulations based on the time-dependent Ginzburg-Landau (tdGL) equations [30-32]. The normalized tdGL equations in the zero electric potential gauge can be written in the following form:

$$
\begin{gathered}
\partial_{t} \psi=(\nabla-i \mathbf{A})^{2} \psi+\left(1-T-|\psi|^{2}\right) \psi, \\
\partial_{t} \mathbf{A}=\operatorname{Re}\left[\psi^{*}(-i \nabla-\mathbf{A}) \psi\right]-\kappa^{2} \nabla \times \nabla \times \mathbf{A},
\end{gathered}
$$

where distances are normalized by the zero-temperature coherence length $\xi(0)$; time by $\xi(0)^{2} / D$, where $D$ is the diffusion coefficient; the order parameter $\psi$ by its value for zero temperature and zero magnetic field; temperature $T$ by $T_{c}$; the magnetic field $H$ by $H_{c 2}=\Phi_{0} / 2 \pi \xi^{2}$; and the vector potential $\mathbf{A}$ by $\Phi_{0} / 2 \pi \xi(0)$. As the thickness of the sample $d \ll \lambda(0)$, the variations of the currents along the thickness can be neglected. In this case, we can apply a two-dimensional model, using an effective coherence length to incorporate the proximity effect of the Au layer on the MoGe film, inducing spatial variations of the order parameter along the thickness of the sample $[8,33]$. The effective penetration depth given by $\Lambda \sim 14 \mu \mathrm{m}$ can be safely assumed to be much larger than the effective coherence length. As such, one can only solve the first equation and simply put $\mathbf{A}=(-H y / 2, H x / 2,0)$ [34], with $H$ corresponding to a spatially homogenous applied magnetic field. The superconductor-vacuum boundary condition $\left.(\boldsymbol{\nabla}-i \mathbf{A}) \psi\right|_{n}=0$ is used for the sample and antidots edges. To reproduce the ZFC procedure in experiments, we start the simulations from the initial state in the absence of the magnetic field at $T=500 \mathrm{mK}$. Subsequently the magnetic field is increased in steps of $5 \mathrm{mT}$, and the vortex states are obtained at each step.

\section{RESULTS AND DISCUSSION}

\section{Mapping the vortex distribution in a mesoscopic square with antidots}

Figure 2(a) shows the topography of sample A obtained by constant current scanning tunneling microscopy, using a bias voltage of $V_{\text {bias }}=5 \mathrm{mV}$ and a fixed tunneling current of $I_{t}=$ $100 \mathrm{pA}$. By comparing with the scanning electron microscopy images in Figure 1(b) (left panel), it is clear that we probe the topography of the top Au layer.

A normalized local tunneling conductance spectrum (normalized to the value at $\left.V_{\text {bias }}=3 \mathrm{mV}\right), d I / d V(V)$, obtained at a center position on the mesoscopic MoGe-Au square at $T=0.5 \mathrm{~K}$ and $H=0 \mathrm{mT}$, is shown in Figure 2(b) and reveals a well-pronounced minigap, indicating the presence of proximity-induced superconducting correlations in the $\mathrm{Au}$ layer $[5,6,8]$. The estimated value of the proximity minigap is $\delta \sim 0.5 \mathrm{meV}$, which gives us a ratio of $\delta \sim 0.55 \Delta$, with $\Delta$ being the bulk gap of our MoGe film as estimated from the critical temperature using the BCS expression. The panels in Fig. 2(c) show a subset of the measured zero-bias conductance (ZBC) maps on sample A obtained after preparing the sample by a zero-field-cooling procedure and increasing subsequently the field in steps of $H=10 \mathrm{mT}$. The dark blue color (high ZBC) implies a high tunneling conductance (normal state), while the yellow color (low ZBC) indicates a low tunneling conductance (the superconducting state). Similarly as in Ref. [20], the ZBC is a sensitive probe to detect the depletion of the superconducting state.

As shown in the first panel of Fig. 2(c), we observe a uniform ZBC map at $H=0 \mathrm{mT}$. The dotted black lines indicate the antidot locations. The depth of the antidots as obtained from the constant current scanning tunneling image [Fig. 2(a)] is $15 \mathrm{~nm}$. This value is considerably less than the designed antidot depth of $30 \mathrm{~nm}$, independently 
confirmed by atomic force microscopy measurements. Henceforth, the low $\mathrm{ZBC}$ value at the antidot locations results from tunneling between the side of the tip and the antidot edge.

Upon increasing the magnetic field, we clearly observe (similar to Ref. [18]) a pronounced minigap suppression in the quasiparticle excitation spectra at the right edge for $H=$ $220 \mathrm{mT}$, together with a weaker suppression at the top and bottom edges. The observed modulation of the condensate can be attributed to a kinetic-induced local depletion of the condensate. This depletion is position dependent due to a current crowding effect induced by edge defects [35] and the reduced current distribution of the supercurrent density at the corners of the square geometry [36-38]. In stark contrast to our earlier investigation [18], performed on uniform islands with similar dimensions at which we observed the first vortex entrance at $H=60 \mathrm{mT}$, we observed the first signature of the presence of a vortex on sample $\mathrm{A}$ at $H=280 \mathrm{mT}$.

Upon increasing the magnetic field beyond $H=280 \mathrm{mT}$, the ZBC maps show clear signatures of interstitial vortices. To indicate the vortex positions we added black solid contour lines in the $\mathrm{ZBC}$ maps, corresponding with a value of $\mathrm{ZBC}=0.83$. At $H=310 \mathrm{mT}$, the first interstitial vortex enters and moves to the center. After the entrance of a first vortex which is located at the center location in between the antidots, a second vortex enters from the right-hand side. Subsequently both vortices shift position, in order to allow a third vortex to enter again from the same preferential entry point. The location of the vortex entry point is in perfect agreement with the observed screening current enhancement at $H=$ $220 \mathrm{mT}$. This confirms that inhomogeneities of the sample edge determine the preferential vortex entry point [18,39]. Upon increasing the field further up to $H=400 \mathrm{mT}$, we obtain a fully developed symmetrical vortex state having a diamond configuration commensurate with the antidot array having C4 symmetry. Despite the fabrication imperfections, the sample quality is high enough to induce a symmetric vortex configuration $[9,11,40]$.

The proximity effect governs the superconducting characteristic length scales in every component of the bilayer nanoislands. As such, it is natural to expect that the proximity effect has an impact on the obtained vortex distributions. In order to investigate this impact, we compare the obtained vortex distributions with detailed simulations of the system. In these simulations we treat the MoGe-Au island as a single layer with an effective coherence length and size. By varying these parameters we can identify the ingredients required to obtain the observed vortex distributions.

A first ingredient is the choice of the effective coherence length. A second ingredient is the effective size of the antidot. Because of wetting issues, the Au hole is effectively bigger than the MoGe hole [see Fig. 1(b)]. Note that it is natural to expect that the effective coherence length will be a weighted average of the coherence length in the MoGe layer and the normal metal coherence length in the Au top layer. To this end, similarly as in Refs. $[8,41]$, the lateral size of the vortex core measured at the Au surface by STS measurements provides a good estimate for the normal metal coherence in the Au layer.
Figure 3(a) presents the normalized ZBC profile along the cross section of a single interstitial vortex. The solid line is a fit using $\sigma_{H}(r)=1-\left(1-\sigma_{0}\right) \tanh r / \xi_{N}$, the phenomenological formula suggested in Ref. [41], in which $\sigma_{0}$ is the ZBC far from the core. The best fit is obtained for $\xi_{N} \sim 40 \mathrm{~nm}$, which exceeds the value in the MoGe layer.

As a starting point for the tdGL simulations, we used $\xi(0) \sim 6.72 \mathrm{~nm}$ (as obtained from transport measurements), corresponding with the coherence length in the MoGe layer and the size of the MoGe antidots [see Fig. 3(b) (left)]. These parameters did not fit the experiments. First, the magnetic field value at which the first interstitial vortex was observed was far below the experimental value. Second, the obtained vortex distributions did not match the experimental images shown in Fig. 2. Both discrepancies indicate that there is significant impact of the gold top layer on the field distributions of vortices in these samples.

To get more realistic simulation results, we increased $\xi$ to incorporate the impact of the gold layer. Nevertheless, this only lowered the magnetic field at which we observed the first vortex. As stated before, it is very reasonable to take an effective antidot size in between the MoGe and gold hole size. We get a very good correspondence with $\xi(0) \sim 10 \mathrm{~nm}$ and an effective antidot size (of $r \sim 69 \mathrm{~nm}$ ) in between the MoGe and Au antidot size. Figure 3(c) presents an overview of the obtained order parameter distributions for magnetic field values similar to those of the experiment shown in Fig. 2. To reproduce the zero-field-cooling procedure in experiments, we start the simulations from the initial state in the absence of the magnetic field at $500 \mathrm{mK}$. Then we subsequently increase the magnetic field to $420 \mathrm{mT}$ in steps of $5 \mathrm{mT}$ and obtain the equilibrium vortex states at each step. The number inside the antidots indicates the winding number, $L$, obtained from the computed phase portraits using tdGl simulations [see Fig. 3(d)]. Upon increasing the field from $H=0 \mathrm{mT}$ to $H=$ $200 \mathrm{mT}$, several vortices enter through the edge of the square and become trapped at the antidot locations [42], effectively increasing the winding number in the four antidots to $L=2$. As illustrated in Fig. 3(e) (left panel), the resulting screening currents at the antidot edges counteract the Meissner currents at the sample edge, reducing the total kinetic energy of the system. Note further that the screening currents at the edge of the antidots are much weaker than the currents at the edge of the square. This explains our inability to observe any manifestation of screening currents at the antidot edge in the ZBC maps within our experimental resolution. Similar to the experimental observations, we observe when ramping up the field further to $H=300 \mathrm{mT}$ the appearance of an interstitial vortex at the central position. The winding number inside each antidot has increased up to $L=4$. This central vortex position is favorable as it results in the lowest kinetic energy of the supercurrent distribution in the sample [Fig. 3(e), right panel]. The circulating vortex currents will counteract the supercurrent surrounding the antidots. Note that at this field value it is energetically more favorable to create a vortex in the condensate instead of increasing the winding number of an antidot, despite the loss of condensation energy necessary to create a vortex. Upon further increasing the field it is the competing interplay between the total kinetic energy and the 
(a)

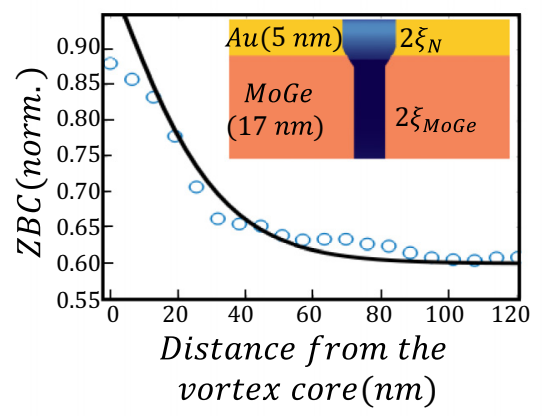

(c)

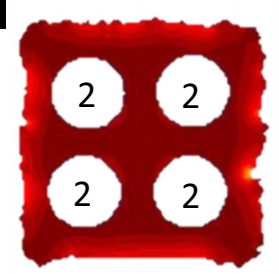

$200 m T$

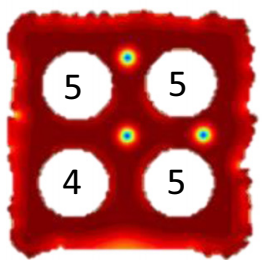

$370 m T$

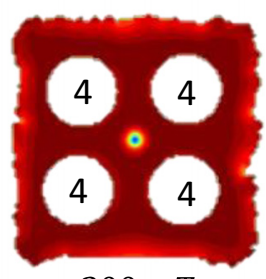

$300 m T$

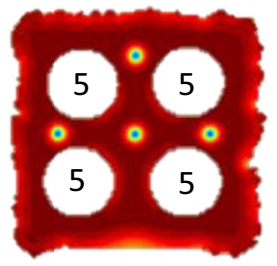

$380 m T$ (b)

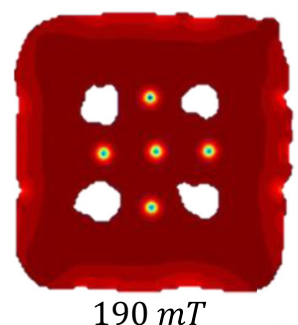

$190 m T$

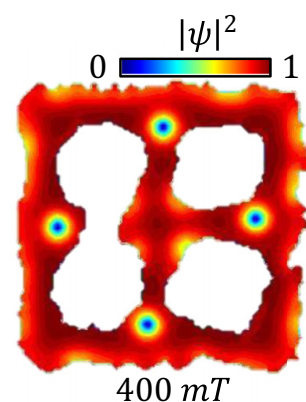

$400 m T$

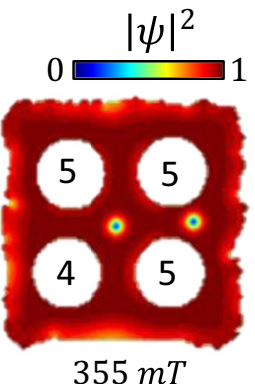

(d)
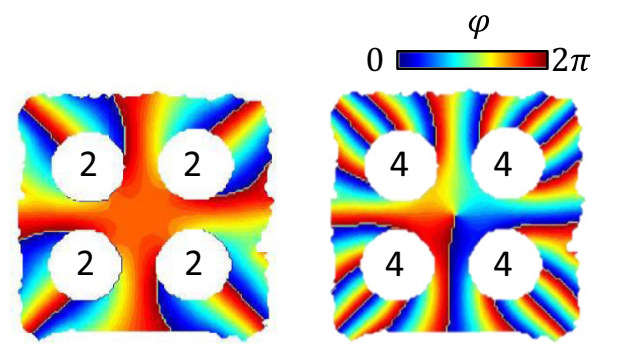

$200 m T$
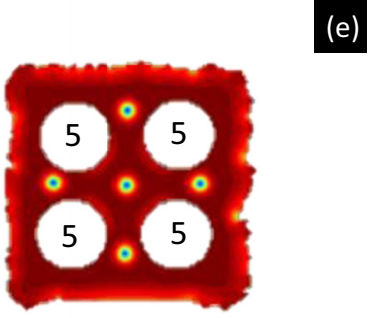

$400 m T$

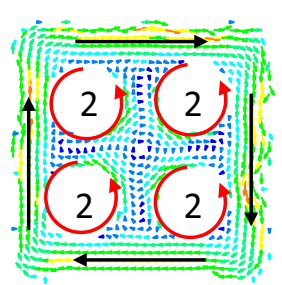

$200 m T$
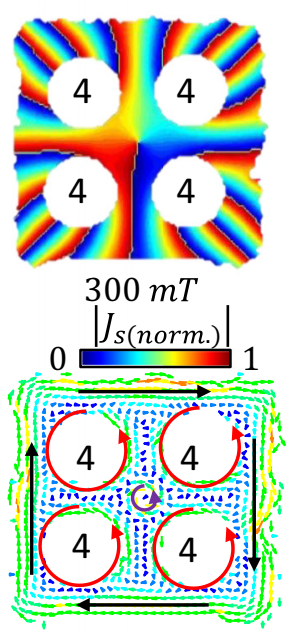

$300 m T$

FIG. 3. (a) The open circles show the normalized ZBC value along a cross section for the vortex observed at a magnetic field of $H=310 \mathrm{mT}$ [see Fig. 2(c)]. The solid black line is a fit as described in the text to extract the effective coherence length in the Au layer. (b) Representation of the square of order parameter obtained via tdGL simulations on sample A at $T=0.5 \mathrm{~K}$ using (left panel) $\xi(0) \sim 6.72 \mathrm{~nm}$ and the size of the MoGe antidots and (right panel) $\xi(0) \sim 18 \mathrm{~nm}$ and the size of the Au edge antidots. (c) The different panels show the magnetic field dependence of the square of the order parameter obtained via tdGL simulations on sample A at $T=0.5 \mathrm{~K}$. The number inside each antidot indicates the winding number, $L$, as calculated from the phase portraits shown in panel (d). (d) The calculated phase portraits $\left(\psi=|\psi| e^{i \phi}\right)$ for sample A at $T=0.5 \mathrm{~K}$ for two magnetic field values. (e) The corresponding supercurrent distribution as obtained via tdGL simulations. The black, red, and blue arrows indicate schematically the Meissner currents, the screening currents of trapped vortices in the antidots, and the screening currents of vortices in the condensate, respectively.

condensation energy for vortex creation that determines the vortex positions [20].

For completeness, we also modeled the vortex distributions when we select the real Au edge [Fig. 3(b), right panel]. In this case we had to increase the coherence length $[\xi(0) \sim 18 \mathrm{~nm}]$ to get the correct magnetic field values at which we observe a certain number of interstitial vortices. The preserved fourfold symmetry of the vortex lattice at $H=380 \mathrm{mT}$ demonstrates that the defect, present in the $\mathrm{Au}$ structure, has no strong influence on the vortex distribution. Nevertheless, a vortex will only appear in the center of the island after the full occupation of all four edges $(H>380 \mathrm{mT})$. This indicates that if the antidots are too large a barrier exists, which prevents vortices from moving to the center. As a consequence, in order to mimic the behavior of the real samples, using twodimensional tdGL simulations, we indeed need to take an average of both the structural and superconducting properties across the bilayer structure.
As demonstrated, the comparison between experiment and theory allows one to identify the different ingredients involved in vortex entry and their distributions at the nanoscale. Using the flexibility of our nanofabrication method, we extended our investigation onto a different sample geometry (sample B) and illustrate once more the one-to-one correspondence between experiment and simulations. This demonstrates that the simple two-dimensional model captures the main ingredients of the bilayer islands. The top left panel in Fig. 4(a) shows the topography of sample B measured by constant current scanning tunneling microscopy, while the remaining panels show the normalized ZBC maps at different magnetic field values. These experimental results exhibit a few particular features which are different in comparison to the results obtained on sample A. First, the increased size of the island is reflected in a lower magnetic field value at which the first interstitial vortex is observed $(H=170 \mathrm{mT})$. Second, the observed vortex distributions have a completely 
(a)

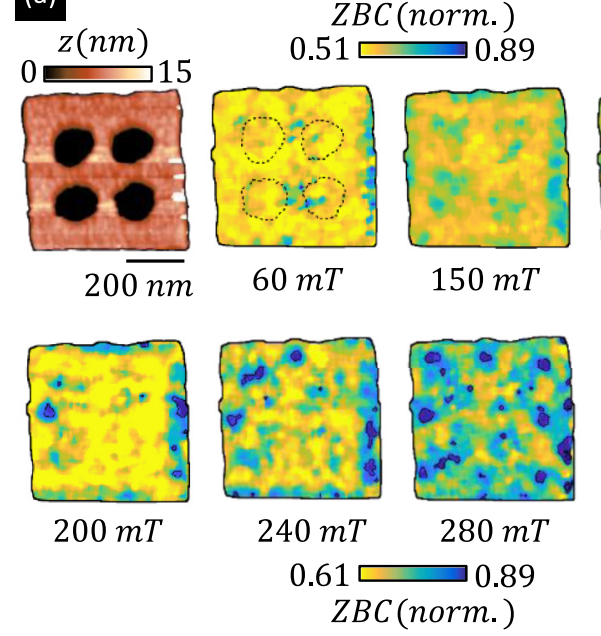

(b)

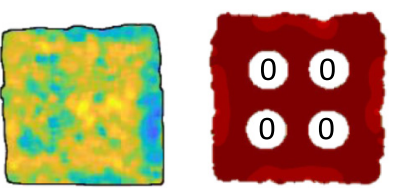

$60 m T$
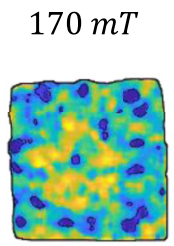

$360 m T$

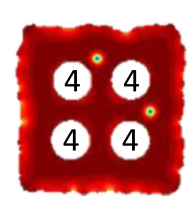

$235 m T$

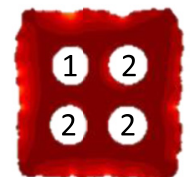

$145 \mathrm{mT}$

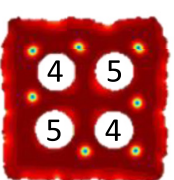

$280 m T$

$0 \longdiv { | \psi | ^ { 2 } } 1$

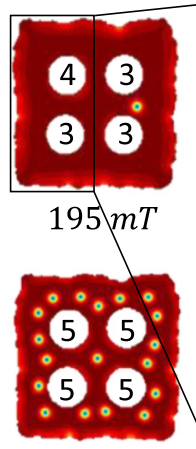

$370 m T$

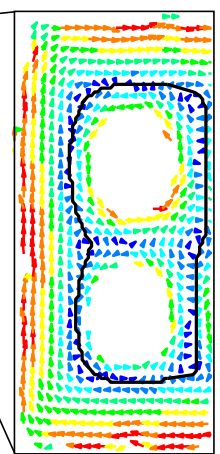

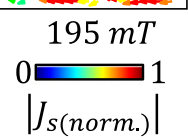

FIG. 4. (a) Top left panel: A constant current topographic STM image of sample B obtained at a fixed tunnel current of $I_{t}=100$ pA, using a bias voltage of $V_{\text {bias }}=5 \mathrm{mV}$. Remaining panels: Normalized ZBC maps obtained by STS measurements (normalized to the value at $V_{\text {bias }}=3 \mathrm{mV}$ ). The different images show a subset of the measured ZBC maps for increasing magnetic field values after preparing the sample by a zero-field-cooling procedure and increasing the field in steps of $H=10 \mathrm{mT}$. Note that the range of the color map for the top three $\mathrm{ZBC}$ maps is different than the range of the color map for the bottom four ZBC maps. The solid black contour lines correspond with a ZBC $=0.83$. The dotted black line in the panel for $H=60 \mathrm{mT}$ indicates the position of the antidots. (b) The panels show the magentic field dependence of the square of the order parameter, numerically generated using the tdGL framework outlined in Sec. IV. The number in each antidot indicates the winding number. The inset (rightmost) figure shows the current distribution map of the highlighted area at $H=195 \mathrm{mT}$. The black line in this current distribution map exhibits the zero-current line as explained in the text.

different character than the vortex distributions observed in Fig. 2.

Figure 4(b) shows the simulated vortex distributions on sample B with the same modeling parameters as used for sample A. In sample A the first vortex is observed in the center of the square, whereas for sample B the first vortices are located in the wider outer rim. This is due to the following size effect. Prior to the entry of the first vortex that resides in the condensate, the Meissner currents in the outer rim oppose the screening currents surrounding the antidots having a nonzero winding number [for example, see Fig. 3(e)]. Whereas in sample A, a zero-supercurrent condition is never established in the thin outer rim surrounding the antidot lattice. The wider rim of sample B results in a zero-current line surrounding the antidot lattice [as indicated by the black solid line in the inset of Fig. 4(b)]. The zero-current line will be a favorable position for the interstitial vortices because at these locations the circulating currents of the vortex will compensate both the Meissner currents at the edge and the screening currents surrounding the antidot, thereby reducing the total kinetic energy. The distribution of the vortices in the outer rim at $H=280 \mathrm{mT}$ follows the symmetry of the antidot lattice. It takes a field value of $H=360 \mathrm{mT}$ in order to observe a vortex in the center of the antidot lattice. At all magnetic fields a very nice correspondence is observed between theory and experiment. This correspondence, once more, confirms that the simple model can capture the main ingredients of the bilayer structure and indicates the need to incorporate the Au layer through an increase of the coherence length and the size of the antidots.

Note that the geometries of samples A and B are good candidates for the stabilization of a symmetry-induced antivortex at the center of the antidot lattice as described in Ref. [43] and yet not resolved by experiment up to date. However, from the calculated phase portraits using the effective tdGL model, we can identify the central vortices in the ZBC maps observed at $H=400 \mathrm{mT}$ [Fig. 2(c)] for sample A and at $H=360 \mathrm{mT}$ [Fig. 4(a)] for sample B to be of vortex nature (no antivortices).

\section{CONCLUSIONS}

To conclude, we performed successfully STS studies on lithographically patterned mesoscopic superconducting squares of a MoGe-Au bilayer containing a square antidot array. Additionally we made a direct comparative study with simulations using the GL framework. We demonstrated that the details of the sample edge (defect centers) and an effective coherence length, capturing the proximity effect in the bilayer, are necessary ingredients to explain the experimentally observed magnetic field dependencies. We believe these findings open the path to perform STS studies on lithographically fabricated superconducting devices and provide insight to optimize their performance and functionalities.

\section{ACKNOWLEDGMENTS}

R.P., B.R, V.V.M., and J.V.d.V. acknowledge support from the Methusalem funding by the Flemish government and the Flemish Science Foundation (Grant No. G0B5315N). C.X. acknowledges support by the National Natural Science Foundation of China (Grant No. 11702218) and the Natural Science Basic Research Plan in Shaanxi Province of China (Grant No. 2018JM1003). This work has been supported by the COST action NanoCoHybri (Grant No. CA16218). 
[1] S. Eley, S. Gopalakrishnan, P. M. Goldbart, and N. Mason, Nat. Phys. 8, 59 (2012).

[2] A. I. Buzdin, Rev. Mod. Phys. 77, 935 (2005).

[3] C. Bøttcher, F. Nichele, M. Kjaergaard, H. Suominen, J. Shabani, C. Palmstrøm, and C. Marcus, Nat. Phys. 14, 1138 (2018).

[4] M. B. Shalom, M. Zhu, V. Fal'Ko, A. Mishchenko, A. Kretinin, K. Novoselov, C. Woods, K. Watanabe, T. Taniguchi, A. Geim, and J. Prance, Nat. Phys. 12, 318 (2016).

[5] W. Belzig, C. Bruder, and G. Schön, Phys. Rev. B 54, 9443 (1996).

[6] M. Vinet, C. Chapelier, and F. Lefloch, Phys. Rev. B 63, 165420 (2001).

[7] S. Pilgram, W. Belzig, and C. Bruder, Phys. B (Amsterdam, Neth.) 280, 442 (2000).

[8] V. S. Stolyarov, T. Cren, C. Brun, I. A. Golovchanskiy, O. V. Skryabina, D. I. Kasatonov, M. M. Khapaev, M. Y. Kupriyanov, A. A. Golubov, and D. Roditchev, Nat. Commun. 9, 2277 (2018).

[9] V. Moshchalkov, L. Gielen, C. Strunk, R. Jonckheere, X. Qiu, C. Van Haesendonck, and Y. Bruynseraede, Nature (London) 373, 319 (1995).

[10] V. V. Moshchalkov, M. Baert, V. V. Metlushko, E. Rosseel, M. J. Van Bael, K. Temst, Y. Bruynseraede, and R. Jonckheere, Phys. Rev. B 57, 3615 (1998).

[11] L. F. Chibotaru, A. Ceulemans, V. Bruyndoncx, and V. V. Moshchalkov, Nature (London) 408, 833 (2000).

[12] A. Oral and S. J. Bending, Appl. Phys. Lett. 69, 1324 (1996).

[13] C. A. Durán, P. L. Gammel, R. E. Miller, and D. J. Bishop, Phys. Rev. B 52, 75 (1995).

[14] A. Bezryadin, Y. N. Ovchinnikov, and B. Pannetier, Phys. Rev. B 53, 8553 (1996).

[15] H. Suderow, M. Crespo, P. Martinez-Samper, J. Rodrigo, G. Rubio-Bollinger, S. Vieira, N. Luchier, J. Brison, and P. Canfield, Phys. C (Amsterdam, Neth.) 369, 106 (2002).

[16] L. Kramer, W. Pesch, and R. Watts-Tobin, Solid State Commun. 14, 1251 (1974).

[17] G. Karapetrov, J. Fedor, M. Iavarone, D. Rosenmann, and W. K. Kwok, Phys. Rev. Lett. 95, 167002 (2005).

[18] M. Timmermans, L. Serrier-Garcia, M. Perini, J. Van de Vondel, and V. V. Moshchalkov, Phys. Rev. B 93, 054514 (2016).

[19] S. J. Bending, Adv. Phys. 48, 449 (1999).

[20] T. Cren, D. Fokin, F. Debontridder, V. Dubost, and D. Roditchev, Phys. Rev. Lett. 102, 127005 (2009).

[21] I. Guillamon, H. Suderow, F. Guinea, and S. Vieira, Phys. Rev. B 77, 134505 (2008).
[22] J. Senzier, P. S. Luo, and H. Courtois, Appl. Phys. Lett. 90, 043114 (2007).

[23] H. le Sueur, P. Joyez, H. Pothier, C. Urbina, and D. Esteve, Phys. Rev. Lett. 100, 197002 (2008).

[24] T. Nishizaki, A. Troyanovski, G. van Baarle, P. Kes, and J. Aarts, Phys. C (Amsterdam, Neth.) 388-389, 777 (2003).

[25] D. Roditchev, C. Brun, L. Serrier-Garcia, J. C. Cuevas, V. H. L. Bessa, M. V. Milošević, F. Debontridder, V. Stolyarov, and T. Cren, Nat. Phys. 11, 332 (2015).

[26] I. Guillamón, H. Suderow, S. Vieira, L. Cario, P. Diener, and P. Rodière, Phys. Rev. Lett. 101, 166407 (2008).

[27] A. K. Gupta, L. Crétinon, N. Moussy, B. Pannetier, and H. Courtois, Phys. Rev. B 69, 104514 (2004).

[28] D. Lotnyk, O. Onufriienko, T. Samuely, O. Shylenko, V. Komanický, P. Szabó, A. Feher, and P. Samuely, Low Temp. Phys. 43, 919 (2017).

[29] M. Motta, F. Colauto, J. I. Vestgården, J. Fritzsche, M. Timmermans, J. Cuppens, C. Attanasio, C. Cirillo, V. V. Moshchalkov, J. Van de Vondel, T. H. Johansen, W. A. Ortiz, and A. V. Silhanek, Phys. Rev. B 89, 134508 (2014).

[30] R. Watts-Tobin, Y. Krähenbühl, and L. Kramer, J. Low Temp. Phys. 42, 459 (1981).

[31] L. Kramer and R. J. Watts-Tobin, Phys. Rev. Lett. 40, 1041 (1978).

[32] M. L. Latimer, G. R. Berdiyorov, Z. L. Xiao, F. M. Peeters, and W. K. Kwok, Phys. Rev. Lett. 111, 067001 (2013).

[33] J. Clarke, J. Phys. Colloq. 29, C2 (1968).

[34] M. L. Latimer, G. R. Berdiyorov, Z. L. Xiao, W. K. Kwok, and F. M. Peeters, Phys. Rev. B 85, 012505 (2012).

[35] J. Gutierrez, B. Raes, J. Van de Vondel, A. V. Silhanek, R. B. G. Kramer, G. W. Ataklti, and V. V. Moshchalkov, Phys. Rev. B 88, 184504 (2013).

[36] B. J. Baelus and F. M. Peeters, Phys. Rev. B 65, 104515 (2002).

[37] B. J. Baelus, A. Kanda, N. Shimizu, K. Tadano, Y. Ootuka, K. Kadowaki, and F. M. Peeters, Phys. Rev. B 73, 024514 (2006).

[38] G. R. Berdiyorov, B. J. Baelus, M. V. Milošević, and F. M. Peeters, Phys. Rev. B 68, 174521 (2003).

[39] L. Serrier-Garcia, M. Timmermans, J. Van de Vondel, and V. V. Moshchalkov, Phys. C (Amsterdam, Neth.) 533, 109 (2017).

[40] T. Puig, E. Rosseel, M. Baert, M. J. Van Bael, V. V. Moshchalkov, and Y. Bruynseraede, Appl. Phys. Lett. 70, 3155 (1997).

[41] M. R. Eskildsen, M. Kugler, S. Tanaka, J. Jun, S. M. Kazakov, J. Karpinski, and O. Fischer, Phys. Rev. Lett. 89, 187003 (2002).

[42] A. I. Buzdin, Phys. Rev. B 47, 11416 (1993).

[43] R. Geurts, M. V. Milošević, and F. M. Peeters, Phys. Rev. B 75, 184511 (2007). 